\title{
AN IMPROVED A PRIORI ERROR ANALYSIS FOR FINITE ELEMENT APPROXIMATIONS OF SIGNORINI'S PROBLEM*
}

\author{
PATRICK HILD ${ }^{\dagger}$ AND YVES RENARD $\ddagger$
}

\begin{abstract}
The present paper is concerned with the unilateral contact model in linear elastostatics, the so-called Signorini problem. (Our results can also be applied to the scalar Signorini problem.) A standard continuous linear finite element approximation is first chosen to approach the two-dimensional problem. We develop a new error analysis in the $H^{1}$-norm using estimates on Poincaré constants with respect to the size of the areas of the noncontact sets. In particular we do not assume any additional hypothesis on the finiteness of the set of transition points between contact and noncontact. This approach allows us to establish better error bounds under sole $H^{\tau}$ assumptions on the solution: if $3 / 2<\tau<2$ we improve the existing rate by a factor $h^{(\tau-3 / 2)^{2}}$ and if $\tau=2$ the existing rate $\left(h^{3 / 4}\right)$ is improved by a new rate of $h \sqrt{|\ln (h)|}$. Using the same finite element spaces as previously we then consider another discrete approximation of the (nonlinear) contact condition in which the same kind of analysis leads to the same convergence rates as for the first approximation.
\end{abstract}

Key words. Signorini problem, unilateral contact, finite elements, a priori error estimates

1. Introduction and notation. Finite element methods are currently used to approximate Signorini's problem or the equivalent scalar valued unilateral problem (see, e.g., $[14,17,18,29,30]$ ). Such a problem shows a nonlinear boundary condition, which roughly speaking requires that (a component of) the solution $u$ is nonpositive (or equivalently nonnegative) on (a part of) the boundary of the domain $\Omega$ (see [25]). This nonlinearity leads to a weak formulation written as a variational inequality which admits a unique solution (see [9]) and the regularity of the solution shows limitations whatever is the regularity of the data (see [21]). A consequence is that only finite element methods of order one and of order two are of interest.

This paper concerns one of the simplest cases: the two-dimensional problem (which corresponds to a nonlinearity holding on a boundary of dimension one) written as a variational inequality and two approximations using continuous conforming linear finite element methods and the corresponding a priori error estimates in the $H^{1}(\Omega)$-norm.

We first consider an approximation in which the discrete convex cone of admissible solutions is a subset of the continuous convex cone of admissible solutions which corresponds to the most common approximation. The existing results concerning the problem can be classified following the regularity assumptions $H^{\tau}(\Omega)$ made on the solution $u$ and following additional assumptions, in particular the hypothesis assuming that there is a finite number of transition points between contact and noncontact. As far as we know, the existing results for this problem can be summarized as follows (we denote by $h$ the discretization parameter) in (E1), (E2), (E3), and (E4):

\footnotetext{
${ }^{\dagger}$ Institut de Mathématiques de Toulouse, CNRS UMR 5219, Université Paul Sabatier, 118 route de Narbonne, 31062 Toulouse Cedex 9, France (phild@math.univ-toulouse.fr).

‡Université de Lyon, CNRS, INSA-Lyon, ICJ UMR5208, LaMCoS UMR5259, F-69621, Villeurbanne, France (Yves.Renard@insa-lyon.fr).
} 
(E1) If $u \in H^{\tau}(\Omega)$ with $1<\tau \leq 3 / 2$, an optimal error estimate of order $h^{\tau-1}$ was obtained in [2].

(E2) If $u \in H^{\tau}(\Omega)$ with $3 / 2<\tau<2$, an analysis as the one in [11, 24] (see also $[13,14])$ leads to a convergence rate of order $h^{\tau / 2-1 / 4}$. Adding the assumption on the finiteness of transition points and using appropriate Sobolev-Morrey inequalities allows us to recover optimality of order $h^{\tau-1}$ (see [2]).

(E3) The case $u \in H^{2}(\Omega)$ is more complicated and requires some technical refinements. The initial analysis in [24] (see also [11, 13, 14]) leads to a convergence rate of order $h^{3 / 4}$. Adding the assumption on the finiteness of transition points has led to the following results and improvements: in [2], the study and the use of the constants $C(q)$ (resp., $C(\alpha)$ ) of the embeddings $H^{1 / 2}(0,1) \rightarrow L^{q}(0,1)$ (resp., $\left.H^{3 / 2}(0,1) \rightarrow C^{0, \alpha}(0,1)\right)$ allows us to obtain a rate of order $h \sqrt{|\ln (h)|}$. The additional use of Gagliardo-Nirenberg inequalities allows us to obtain a slightly better rate of order $h \sqrt[4]{|\ln (h)|}$ in [3]. Finally a different analysis using an additional modified Lagrange interpolation operator and fine estimates of the solution near the (finite number of) transition points had led to optimality of order $h$ in [16].

(E4) If $u \in H^{\tau}(\Omega)$ with $\tau>2$ the analysis in [11] shows that convergence of order $h$ is obtained when $\tau=5 / 2$ (more precisely if the solution lies in $H^{2}(\Omega)$ and its trace lies in $\left.H^{2}(\partial \Omega)\right)$. Similar assumptions are used in [4] to obtain the convergence of order $h$. Recently, in [23] the use of the Peetre-Tartar lemma (see $[22,26,27,7]$ ) has led to an analysis which requires only $H^{2+\varepsilon}(\Omega)$ regularity $(\varepsilon>0)$ to obtain a convergence of order $h$.

We assume in this paper $H^{\tau}(\Omega)$ regularity $(3 / 2<\tau \leq 2)$ for $u$ without any additional assumption (in particular those concerning the finiteness of the set of transition points). In this case the existing error bound is $h^{\tau / 2-1 / 4}$. We develop a new analysis which consists of classifying the finite elements on the contact zone into two cases. A first case where the unknown vanishes near both extremities of the segment and the other case where the dual unknown (the normal derivative for the scalar Signorini problem and the normal constraint for the unilateral contact problem) vanishes on an area near a segment extremity. We then study for various fractional Sobolev spaces the behavior of the constants $C(\theta)$ occurring in Poincaré inequalities with respect to the length $\theta$ of the area where the unknown vanishes. This analysis leads to the following new results denoted by (N1) and (N2):

(N1) If $u \in H^{\tau}(\Omega)$ with $3 / 2<\tau<2$ we obtain a convergence rate of order $h^{\tau / 2-1 / 4+(\tau-3 / 2)^{2}}$ which improves the existing rate of $h^{\tau / 2-1 / 4}$. Note that the convergence rate becomes optimal when $\tau \rightarrow 3 / 2,(\tau>3 / 2)$ and when $\tau \rightarrow 2,(\tau<2)$. The regularity where we are less close to optimality is when $\tau=7 / 4$, where we obtain a rate of $h^{11 / 16}$, whereas optimality is $h^{3 / 4}$. So the maximal distance to optimality is $h^{1 / 16}$ (see Figure 3.1).

(N2) If $u \in H^{2}(\Omega)$ we obtain a quasi-optimal convergence rate of order $h \sqrt{|\ln (h)|}$ which improves the existing rate of $h^{3 / 4}$.

We also consider in this paper a second finite element approximation in which the discrete convex cone of admissible functions is not a subset of the continuous convex cone of admissible functions. In this case fewer results available as for the first approximation. In particular the results in (E3) are available $\left(h^{3 / 4}\right.$ error bound) without additional assumption on the contact set (see $[14,19])$. For a slightly different approach (using quadratic finite elements), [3] obtains under $H^{2}$ regularity an error bound of $h^{3 / 4}$ and of $h \sqrt[4]{|\ln (h)|}$ with an additional assumption on the finiteness of the transition points. Note that the results in (E2) without additional assumption on the 
contact set could be easily obtained using the techniques in the above references. The use of an adaption of our technique allows us to recover for this second approximation the results (N1), and (N2) and the result in (E4) of [23].

We next give a comment concerning the finiteness of the set of transition points. From a practical viewpoint, one may think that the assumption of finiteness on the number of transition points between contact and noncontact is always satisfied, apart from very specific situations. Even if this question has not been solved theoretically, some evidence suggests that it could not be the case. Indeed, when considering on a straight edge a transition from a Dirichlet boundary condition to a Neumann boundary condition, the asymptotic displacement which appears near the transition is infinitely oscillating with (for instance) a dependence in $\sin (\ln (r)$ ), where $r$ is the distance to the transition point (see [10]). Thus, paradoxically, in the case of the Dirichlet-Signorini transition, one can imagine that there is always the presence of contact close to the transition point, whether the structure is pushed to promote contact or, on the contrary, when it is pulled in the direction of separation. This counterintuitive example may bring us to think that the real contact area can be complex even in simple situations. Real contact areas of fractal type cannot a priori be excluded either.

The paper is organized as follows. Section 2 deals with the formulation of the problem, its associated weak form written as a variational inequality, and the most common approximation using the standard continuous linear finite element method. In section 3, we achieve a new error analysis for this method to improve the existing results. Section 4 deals again with the standard continuous linear finite element method, but another approximation of the convex set of admissible displacements is chosen. All the results of section 3 can be generalized to this case. Two appendices concerning the estimates of Poincaré constants and some interpolation error estimates in fractional Sobolev spaces terminate the paper.

Next, we specify some notation we shall use. Let a Lipschitz domain $\Omega \subset \mathbb{R}^{2}$ be given; the generic point of $\Omega$ is denoted by $x$. The classical Lebesgue space $L^{p}(\Omega)$ is endowed with the norm

$$
\|\psi\|_{L^{p}(\Omega)}=\left(\int_{\Omega}|\psi(x)|^{p} d x\right)^{\frac{1}{p}} .
$$

We will make a constant use of the standard Sobolev space $H^{m}(\Omega), m \geq 0$ (we adopt the convention $H^{0}(\Omega)=L^{2}(\Omega)$ ), provided with the norm

$$
\|\psi\|_{m, \Omega}=\left(\sum_{0 \leq|\alpha| \leq m}\left\|\partial^{\alpha} \psi\right\|_{L^{2}(\Omega)}^{2}\right)^{\frac{1}{2}}
$$

where $\alpha=\left(\alpha_{1}, \alpha_{2}\right)$ is a multi-index in $\mathbb{N}^{2}$ and the symbol $\partial^{\alpha}$ represents a partial derivative. The fractional Sobolev space $H^{\tau}(\Omega), \tau \in \mathbb{R}_{+} \backslash \mathbb{N}$, is defined by the norm $($ see $[1,10])$

$$
\begin{aligned}
\|\psi\|_{\tau, \Omega} & =\left(\|\psi\|_{m, \Omega}^{2}+\sum_{|\alpha|=m} \int_{\Omega} \int_{\Omega} \frac{\left(\partial^{\alpha} \psi(x)-\partial^{\alpha} \psi(y)\right)^{2}}{|x-y|^{2+2 \nu}} d x d y\right)^{\frac{1}{2}} \\
& =\left(\|\psi\|_{m, \Omega}^{2}+\sum_{|\alpha|=m}\left|\partial^{\alpha} \psi\right|_{\nu, \Omega}^{2}\right)^{\frac{1}{2}}
\end{aligned}
$$




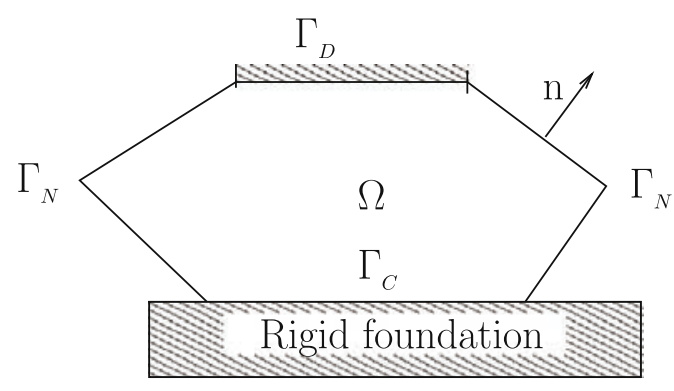

FIG. 2.1. Elastic body $\Omega$ in contact.

where $\tau=m+\nu, m$ being the integer part of $\tau$ and $\nu \in(0,1)$.

For simplicity, to avoid dealing with a nonconformity coming from the approximation of the domain, we shall only consider here polygonally shaped domains. The boundary $\partial \Omega$ is the union of a finite number of segments $\Gamma_{j}, 0 \leq j \leq J$. In such a case, the space $H^{\tau}(\Omega)$ defined above coincides not only with the set of restrictions to $\Omega$ of all functions of $H^{\tau}\left(\mathbb{R}^{2}\right.$ ) (see [10]) but also with the Sobolev space defined by Hilbertian interpolation of standard spaces $\left(H^{m}(\Omega)\right)_{m \in \mathbb{N}}$ and the norms resulting from the different definitions of $H^{\tau}(\Omega)$ are equivalent (see [28]).

To handle trace functions we introduce, for any $\tau \in \mathbb{R}_{+} \backslash \mathbb{N}$, the Hilbert space $H^{\tau}\left(\Gamma_{j}\right)$ associated with the norm

$$
\begin{aligned}
\|\psi\|_{\tau, \Gamma_{j}} & =\left(\|\psi\|_{m, \Gamma_{j}}^{2}+\int_{\Gamma_{j}} \int_{\Gamma_{j}} \frac{\left(\psi^{(m)}(x)-\psi^{(m)}(y)\right)^{2}}{|x-y|^{1+2 \nu}} d \Gamma d \Gamma\right)^{\frac{1}{2}} \\
& =\left(\|\psi\|_{m, \Gamma_{j}}^{2}+\left|\psi^{(m)}\right|_{\nu, \Gamma_{j}}^{2}\right)^{\frac{1}{2}},
\end{aligned}
$$

where $m$ is the integer part of $\tau$ and $\nu$ stands for its decimal part. Finally the trace operator $T: \psi \mapsto\left(\psi_{\mid \Gamma_{j}}\right)_{1 \leq j \leq J}$ maps continuously $H^{\tau}(\Omega)$ onto $\prod_{j=1}^{J} H^{\tau-1 / 2}\left(\Gamma_{j}\right)$ when $\tau>1 / 2$ (see, e.g., [20]).

\section{Signorini's problem and its finite element discretization.}

2.1. Setting of the problem. Let $\Omega \subset \mathbb{R}^{2}$ be a polygonal domain representing the reference configuration of a linearly elastic body whose boundary $\partial \Omega$ consists of three nonoverlapping open parts $\Gamma_{N}, \Gamma_{D}$, and $\Gamma_{C}$ with $\overline{\Gamma_{N}} \cup \overline{\Gamma_{D}} \cup \overline{\Gamma_{C}}=\partial \Omega$. We assume that the measures of $\Gamma_{C}$ and $\Gamma_{D}$ in $\partial \Omega$ are positive and, in order to simplify, that $\Gamma_{C}$ is a straight line segment. The body is submitted to a Neumann condition on $\Gamma_{N}$ with a density of loads $F \in\left(L^{2}\left(\Gamma_{N}\right)\right)^{2}$, a Dirichlet condition on $\Gamma_{D}$ (the body is assumed to be clamped on $\Gamma_{D}$ to simplify), and to volume loads denoted by $f \in\left(L^{2}(\Omega)\right)^{2}$ in $\Omega$. Finally, a (frictionless) unilateral contact condition between the body and a flat rigid foundation holds on $\Gamma_{C}$ (see Figure 2.1). The problem consists in finding the displacement field $u: \bar{\Omega} \rightarrow \mathbb{R}^{2}$ satisfying (2.1)-(2.6):

$$
\begin{aligned}
-\operatorname{div} \sigma(u)=f & \text { in } \Omega, \\
\sigma(u)=\mathcal{A} \varepsilon(u) & \text { in } \Omega, \\
\sigma(u) \mathrm{n}=F & \text { on } \Gamma_{N}, \\
u=0 & \text { on } \Gamma_{D},
\end{aligned}
$$

where $\sigma(u)$ represents the stress tensor field, $\varepsilon(u)=\left(\nabla u+(\nabla u)^{T}\right) / 2$ denotes the linearized strain tensor field, $\mathrm{n}$ stands for the outward unit normal to $\Omega$ on $\partial \Omega$, and 
$\mathcal{A}$ is the fourth order elastic coefficient tensor which satisfies the usual symmetry and ellipticity conditions and whose components are in $L^{\infty}(\Omega)$.

On $\Gamma_{C}$, we decompose the displacement and the stress vector fields in normal and tangential components as follows:

$$
\begin{gathered}
u_{N}=u \cdot \mathrm{n}, \quad u_{T}=u-u_{N} \mathrm{n}, \\
\sigma_{N}=(\sigma(u) \mathrm{n}) \cdot \mathrm{n}, \quad \sigma_{T}=\sigma(u) \mathrm{n}-\sigma_{N} \mathrm{n} .
\end{gathered}
$$

The unilateral contact condition on $\Gamma_{C}$ is expressed by the complementary condition

$$
u_{N} \leq 0, \sigma_{N} \leq 0, u_{N} \sigma_{N}=0,
$$

where a vanishing gap between the elastic solid and the rigid foundation has been chosen in the reference configuration.

The frictionless condition on $\Gamma_{C}$ reads as

$$
\sigma_{T}=0 .
$$

Remark 1. This problem is the vector valued version of the scalar Signorini problem which (written in its simplest form) consists of finding the field $u: \bar{\Omega} \rightarrow \mathbb{R}$ satisfying

$$
-\Delta u+u=f \text { in } \Omega, u \leq 0, \frac{\partial u}{\partial n} \leq 0, u \frac{\partial u}{\partial n}=0 \text { on } \partial \Omega .
$$

All the results proved in this paper, in particular, the error estimates in Theorem 3.1 and Theorem 4.1, can be straightforwardly extended to the scalar Signorini problem.

Let us introduce the following Hilbert space:

$$
V=\left\{v \in\left(H^{1}(\Omega)\right)^{2}: v=0 \text { on } \Gamma_{D}\right\} .
$$

The set of admissible displacements satisfying the noninterpenetration conditions on the contact zone is

$$
K=\left\{v \in V: v_{N}=v \cdot n \leq 0 \text { on } \Gamma_{C}\right\} .
$$

Let be given the following forms for any $u$ and $v$ in $V$ :

$$
\begin{gathered}
a(u, v)=\int_{\Omega} \mathcal{A} \varepsilon(u): \varepsilon(v) d \Omega, \\
l(v)=\int_{\Omega} f \cdot v d \Omega+\int_{\Gamma_{N}} F \cdot v d \Gamma,
\end{gathered}
$$

which represent the virtual work of the elastic forces and of the external loads, respectively. From the previous assumptions it follows that $a(\cdot, \cdot)$ is a bilinear symmetric $V$-elliptic and continuous form on $V \times V$ and $l$ is a linear continuous form on $V$.

The weak formulation of problem (2.1)-(2.6) (written as an inequality), introduced in [9] (see also, e.g., [12, 14, 17]), is

$$
\left\{\begin{array}{l}
\text { Find } u \in K \text { satisfying } \\
a(u, v-u) \geq l(v-u) \quad \forall v \in K .
\end{array}\right.
$$

Problem (2.7) admits a unique solution according to Stampacchia's theorem. 
2.2. The standard finite element approximation. Let $V^{h} \subset V$ be a family of finite-dimensional vector spaces indexed by $h$ coming from a regular family $T^{h}$ (see [5]) of triangulations of the domain $\Omega$. The notation $h$ represents the largest diameter among all elements $T \in T^{h}$ which are supposed closed. We choose standard continuous and piecewise affine functions, i.e.,

$$
V^{h}=\left\{v^{h} \in(C(\bar{\Omega}))^{2}:\left.v^{h}\right|_{T} \in P_{1}(T) \forall T \in T^{h}, v^{h}=0 \text { on } \Gamma_{D}\right\} .
$$

The discrete set of admissible displacements satisfying the noninterpenetration conditions on the contact zone is given by

$$
K^{h}=\left\{v^{h} \in V^{h}: v_{N}^{h} \leq 0 \quad \text { on } \Gamma_{C}\right\} .
$$

The discrete variational inequality issued from $(2.7)$ is

$$
\left\{\begin{array}{l}
\text { Find } u^{h} \in K^{h} \text { satisfying } \\
a\left(u^{h}, v^{h}-u^{h}\right) \geq l\left(v^{h}-u^{h}\right) \quad \forall v^{h} \in K^{h} .
\end{array}\right.
$$

According to Stampacchia's theorem, problem (2.9) admits also a unique solution.

3. Error analysis. The forthcoming theorem gives a priori error estimates and is divided into two parts: a first part where the regularity of $u$ is assumed to lie strictly between $H^{3 / 2}(\Omega)$ and $H^{2}(\Omega)$ and a second part in which the $H^{2}(\Omega)$-regularity is considered separately. Afterward, we denote by $C$ a positive generic constant which depends on neither the mesh size $h$ nor the solution $u$.

TheOREm 3.1. Let $u$ and $u^{h}$ be the solutions to problems (2.7) and (2.9), respectively.

Assume that $u \in\left(H^{\tau}(\Omega)\right)^{2}$ with $3 / 2<\tau<2$. Then, there exists a constant $C>0$ independent of $h$ and $u$ such that

$$
\left\|u-u^{h}\right\|_{1, \Omega} \leq C h^{\tau^{2}-\frac{5}{2} \tau+2}\|u\|_{\tau, \Omega} .
$$

Assume that $u \in\left(H^{2}(\Omega)\right)^{2}$. Then, there exists a constant $C>0$ independent of $h$ and $u$ such that

$$
\left\|u-u^{h}\right\|_{1, \Omega} \leq C h \sqrt{|\ln (h)|}\|u\|_{2, \Omega} .
$$

The curve of the new rate in Theorem 3.1 (as a function of the Sobolev exponent $\tau)$, which is compared to the existing one and to the optimal one, is depicted in Figure 3.1.

Remark 2. Unlike some other problems governed by variational inequalities, the location of the nonlinearity in Signorini's problem is in the boundary conditions. When using the standard approach issued from Falk's lemma [8], the inequalities in the boundary conditions require the handling of dual Sobolev norms (i.e., when $u \in H^{2}(\Omega)$ the estimate of $\left\|u_{N}-\left(\mathcal{I}^{h} u\right)_{N}\right\|_{1 / 2, *, \Gamma_{C}}$, where $\|\cdot\|_{1 / 2, *, \Gamma_{C}}$ stands for the dual norm of $\|\cdot\|_{1 / 2, \Gamma_{C}}$ and where $\mathcal{I}^{h}$ denotes the Lagrange interpolation operator mapping into $V^{h}$ ). As already mentioned in the early analysis of [24], better bounds than $h^{3 / 4}$ were not available. In [15] counterexamples were given which confirm that better bounds could not be obtained when estimating $\left\|u_{N}-\left(\mathcal{I}^{h} u\right)_{N}\right\|_{1 / 2, *, \Gamma_{C}}$. As a consequence other techniques must be developed.

Remark 3. Actually, we are not able to extend successfully the results of the theorem to the three-dimensional case since the estimates of the Poincaré constants 


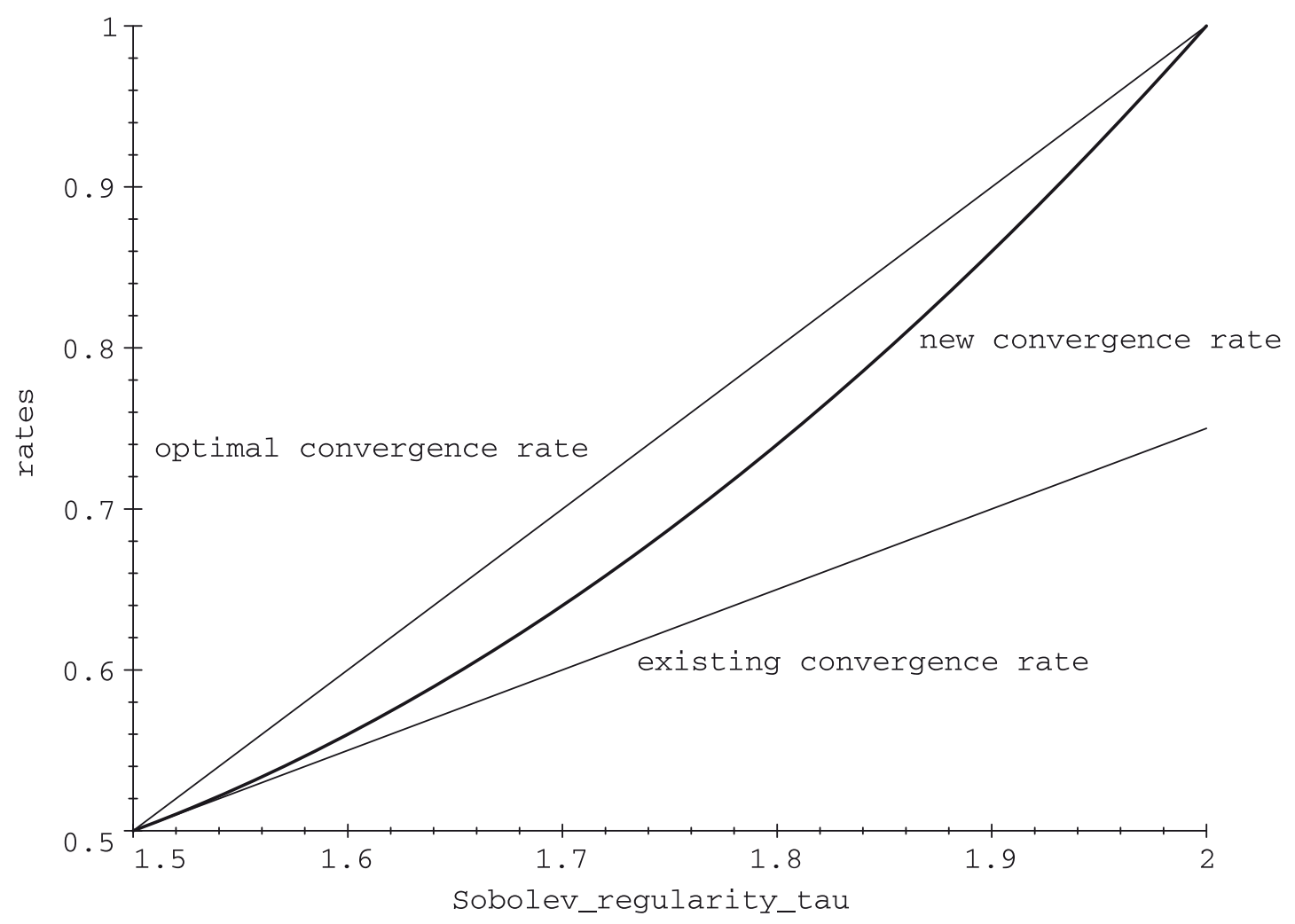

FIG. 3.1. Convergence rates: the existing ones, the ones obtained in this paper, and the optimal ones.

(in Lemma 5.2) are different and do not lead to improved convergence rates. This question remains nevertheless under investigation. In the same way, the extension of the technique to improve the existing results obtained when using quadratic finite element methods could be interesting.

Proof. The use of Falk's lemma (see [8] for the early idea and, e.g., [24, 17, 14] for the adaption to contact problems) leads to the following bound:

$$
\left\|u-u^{h}\right\|_{1, \Omega}^{2} \leq C \inf _{v^{h} \in K^{h}}\left(\left\|u-v^{h}\right\|_{1, \Omega}^{2}+\int_{\Gamma_{C}} \sigma_{N}\left(v^{h}-u\right)_{N} d \Gamma\right),
$$

where $C$ is a positive constant which only depends on the continuity and the ellipticity constants of $a(. .$.$) . The usual choice for v^{h}$ (which we also adopt in this study) is $v^{h}=\mathcal{I}^{h} u$, where $\mathcal{I}^{h}$ is the Lagrange interpolation operator mapping onto $V^{h}$. Of course, $\mathcal{I}^{h} u \in K^{h}$ and $\left\|u-\mathcal{I}^{h} u\right\|_{1, \Omega} \leq C h^{\tau-1}\|u\|_{\tau, \Omega}$ for any $1<\tau \leq 2$.

To prove the theorem it remains then to estimate the term

$$
\int_{\Gamma_{C}} \sigma_{N}\left(\mathcal{I}^{h} u\right)_{N} d \Gamma
$$

for $u \in\left(H^{\tau}(\Omega)\right)^{2}, 3 / 2<\tau \leq 2$. From the trace theorem we deduce that $u_{N} \in$ $H^{\tau-1 / 2}\left(\Gamma_{C}\right)$ (hence $u_{N}$ is continuous) and $\sigma_{N} \in H^{\tau-3 / 2}\left(\Gamma_{C}\right)$. Let $T \in T^{h}$ with $T \cap \Gamma_{C} \neq \emptyset$. In the forthcoming proof we will estimate

$$
\int_{T \cap \Gamma_{C}} \sigma_{N}\left(\mathcal{I}^{h} u\right)_{N} d \Gamma
$$

and we will denote by $h_{e}$ the length of the segment $T \cap \Gamma_{C}$. 


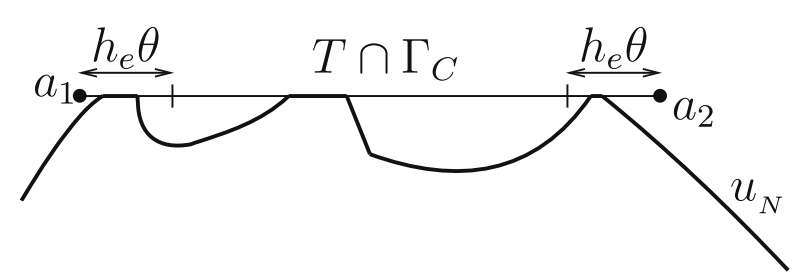

First case: $u_{N}$ vanishes near each vertex.



Second case: $\sigma_{N}$ vanishes on a segment of length $h_{e} \theta$ containing a vertex.

FIG. 3.2. The alternative: first and second cases.

Let $0<\theta<1$ be fixed (the optimal choice of $\theta$ will be done later) and let $T \in T^{h}$ be an element with $T \cap \Gamma_{C} \neq \emptyset$. We will consider the following alternative which is an important point of our analysis:

First case: for any of the two vertices of $T \cap \Gamma_{C}$ there exists a point where $u_{N}$ vanishes at a distance less than $h_{e} \theta$ to a vertex of $T \cap \Gamma_{C}$.

Second case: the normal stress $\sigma_{N}$ vanishes on a segment of length $h_{e} \theta$ including one of the two vertices.

Note that any of the straight line segments $T \cap \Gamma_{C}$ satisfy (at least) one of both previous cases because of the complementarity condition $\sigma_{N} u_{N}=0$ satisfied on $\Gamma_{C}$. The alternative is depicted in Figure 3.2.

First case. Let us denote by $a_{1}, a_{2}$ the two vertices of $T \cap \Gamma_{C}$. There exist $\xi_{1}, \xi_{2} \in T \cap \overline{\Gamma_{C}}$ such that $u_{N}\left(\xi_{1}\right)=u_{N}\left(\xi_{2}\right)=0$ and $\left|a_{i}-\xi_{i}\right| \leq h_{e} \theta, i=1,2$ (where $\left.h_{e}=\left|a_{2}-a_{1}\right|\right)$. Then

$$
\begin{aligned}
\int_{T \cap \Gamma_{C}} \sigma_{N}\left(\mathcal{I}^{h} u\right)_{N} d \Gamma & \leq\left\|\sigma_{N}\right\|_{0, T \cap \Gamma_{C}}\left\|\left(\mathcal{I}^{h} u\right)_{N}\right\|_{0, T \cap \Gamma_{C}} \\
& \leq\left\|\sigma_{N}\right\|_{0, T \cap \Gamma_{C}} h_{e}^{1 / 2} \max \left(\left|u_{N}\left(a_{1}\right)\right|,\left|u_{N}\left(a_{2}\right)\right|\right) .
\end{aligned}
$$

Moreover, for $i=1,2$,

$$
\left|u_{N}\left(a_{i}\right)\right|=\left|u_{N}\left(a_{i}\right)-u_{N}\left(\xi_{i}\right)\right|=\left|\int_{a_{i}}^{\xi_{i}} u_{N}^{\prime}(\zeta) d \zeta\right|,
$$

where $u_{N}^{\prime}$ denotes the derivative along the line $\left(a_{1}, a_{2}\right)$. Suppose now that $\tau \in(3 / 2,2)$ (the case $\tau=2$ is handled next). We set $q=1 /(2-\tau)$ (so $q \in(2,+\infty))$ and one obtains, thanks to the Hölder inequality,

$$
\left|\int_{a_{i}}^{\xi_{i}} u_{N}^{\prime}(\zeta) d \zeta\right| \leq\left(h_{e} \theta\right)^{\tau-1}\left\|u_{N}^{\prime}\right\|_{L^{q}\left(T \cap \Gamma_{C}\right)} .
$$

Now, we can use the continuous embedding of $H^{\tau-3 / 2}\left(T \cap \Gamma_{C}\right)$ into $L^{q}\left(T \cap \Gamma_{C}\right)$ (see, e.g., [1]). In order to obtain a continuity constant independent of the element size, we use the reference element $\tilde{I}=(0,1)$ and we denote $\tilde{u}_{N}(\tilde{x})=u_{N}(\eta(\tilde{x}))$, where $\eta: \overline{\tilde{I}} \rightarrow \overline{T \cap \Gamma_{C}}$ is an affine transformation. We obtain

$$
\left\|u_{N}^{\prime}\right\|_{L^{q}\left(T \cap \Gamma_{C}\right)}=h_{e}^{1 / q}\left\|\tilde{u}_{N}^{\prime}\right\|_{L^{q}(\tilde{I})}=h_{e}^{2-\tau}\left\|\tilde{u}_{N}^{\prime}\right\|_{L^{q}(\tilde{I})} \leq C h_{e}^{2-\tau}\left\|\tilde{u}_{N}^{\prime}\right\|_{\tau-3 / 2, \tilde{I}} .
$$


It remains to bound $\left\|\tilde{u}_{N}^{\prime}\right\|_{\tau-3 / 2, \tilde{I}}$. From the definition of the norms and the affine transformation, we get

$$
\begin{aligned}
\left\|\tilde{u}_{N}^{\prime}\right\|_{\tau-3 / 2, \tilde{I}} & \leq\left\|\tilde{u}_{N}^{\prime}\right\|_{0, \tilde{I}}+\left|\tilde{u}_{N}^{\prime}\right|_{\tau-3 / 2, \tilde{I}} \\
& =h_{e}^{-1 / 2}\left\|u_{N}^{\prime}\right\|_{0, T \cap \Gamma_{C}}+h_{e}^{\tau-2}\left|u_{N}^{\prime}\right|_{\tau-3 / 2, T \cap \Gamma_{C}} \\
& \leq C h_{e}^{-1 / 2}\left\|u_{N}^{\prime}\right\|_{\tau-3 / 2, T \cap \Gamma_{C}} .
\end{aligned}
$$

From (3.6) and (3.7), we deduce

$$
\left\|u_{N}^{\prime}\right\|_{L^{q}\left(T \cap \Gamma_{C}\right)} \leq C h_{e}^{3 / 2-\tau}\left\|u_{N}^{\prime}\right\|_{\tau-3 / 2, T \cap \Gamma_{C}} .
$$

Combining estimates (3.3), (3.4), (3.5), and (3.8) and Young's inequality, we obtain the estimate

$$
\begin{aligned}
\int_{T \cap \Gamma_{C}} \sigma_{N}\left(\mathcal{I}^{h} u\right)_{N} d \Gamma & \leq C h_{e} \theta^{\tau-1}\left\|\sigma_{N}\right\|_{0, T \cap \Gamma_{C}}\left\|u_{N}^{\prime}\right\|_{\tau-3 / 2, T \cap \Gamma_{C}} \\
& \leq C h_{e} \theta^{\tau-1}\left(\left\|\sigma_{N}\right\|_{\tau-3 / 2, T \cap \Gamma_{C}}^{2}+\left\|u_{N}\right\|_{\tau-1 / 2, T \cap \Gamma_{C}}^{2}\right) .
\end{aligned}
$$

The case $\tau=2$ has to be handled separately since $H^{1 / 2}\left(T \cap \Gamma_{C}\right) \not \subset L^{\infty}\left(T \cap \Gamma_{C}\right)$. Using for any $q \in(1,+\infty)$ the continuous embedding of $H^{1 / 2}\left(T \cap \Gamma_{C}\right)$ into $L^{q}\left(T \cap \Gamma_{C}\right)$ (see, e.g., [1]) and the same approach as in (3.5)-(3.9), we get for any $q \in(1,+\infty)$

$$
\int_{T \cap \Gamma_{C}} \sigma_{N}\left(\mathcal{I}^{h} u\right)_{N} d \Gamma \leq C(q) h_{e} \theta^{1-\frac{1}{q}}\left(\left\|\sigma_{N}\right\|_{1 / 2, T \cap \Gamma_{C}}^{2}+\left\|u_{N}\right\|_{3 / 2, T \cap \Gamma_{C}}^{2}\right) .
$$

Second case. Otherwise, by the complementarity condition, $\sigma_{N}$ vanishes on an interval of length $h_{e} \theta$ included in $T \cap \Gamma_{C}$ and having one of the two vertices of $T \cap \Gamma_{C}$ as an extremity. We make the following estimate:

$$
\begin{aligned}
\int_{T \cap \Gamma_{C}} \sigma_{N}\left(\mathcal{I}^{h} u\right)_{N} d \Gamma & =\int_{T \cap \Gamma_{C}} \sigma_{N}\left(\left(\mathcal{I}^{h} u\right)_{N}-u_{N}\right) d \Gamma \\
& \leq\left\|\sigma_{N}\right\|_{0, T \cap \Gamma_{C}}\left\|\left(\mathcal{I}^{h} u\right)_{N}-u_{N}\right\|_{0, T \cap \Gamma_{C}} .
\end{aligned}
$$

Suppose now that $\tau \in(3 / 2,2)$ (the case $\tau=2$ is handled next). On the one hand, by passing on the reference element and applying (5.1) in Lemma 5.2 we obtain

$$
\begin{aligned}
\left\|\sigma_{N}\right\|_{0, T \cap \Gamma_{C}}=h_{e}^{1 / 2}\left\|\tilde{\sigma}_{N}\right\|_{0, \tilde{I}} & \leq C h_{e}^{1 / 2} \theta^{\tau-2}\left|\tilde{\sigma}_{N}\right|_{\tau-3 / 2, \tilde{I}} \\
& =C h_{e}^{\tau-3 / 2} \theta^{\tau-2}\left|\sigma_{N}\right|_{\tau-3 / 2, T \cap \Gamma_{C}} .
\end{aligned}
$$

On the other hand, Lemma 6.1 gives the following estimate:

$$
\left\|\left(\mathcal{I}^{h} u\right)_{N}-u_{N}\right\|_{0, T \cap \Gamma_{C}} \leq h_{e}^{\tau-1 / 2}\left|u_{N}\right|_{\tau-1 / 2, T \cap \Gamma_{C}} .
$$

Thanks to (3.11), (3.12), and (3.13), we get

$$
\begin{aligned}
\int_{T \cap \Gamma_{C}} \sigma_{N}\left(\mathcal{I}^{h} u\right)_{N} d \Gamma & \leq C h_{e}^{2 \tau-2} \theta^{\tau-2}\left|\sigma_{N}\right|_{\tau-3 / 2, T \cap \Gamma_{C}}\left|u_{N}\right|_{\tau-1 / 2, T \cap \Gamma_{C}} \\
& \leq C h_{e}^{2 \tau-2} \theta^{\tau-2}\left(\left\|\sigma_{N}\right\|_{\tau-3 / 2, T \cap \Gamma_{C}}^{2}+\left\|u_{N}\right\|_{\tau-1 / 2, T \cap \Gamma_{C}}^{2}\right) .
\end{aligned}
$$


When $\tau=2$, we achieve the same calculations as in (3.11)-(3.14) by using (5.2) in Lemma 5.2. We get

$$
\int_{T \cap \Gamma_{C}} \sigma_{N}\left(\mathcal{I}^{h} u\right)_{N} d \Gamma \leq C h_{e}^{2} \ln (1 / \theta)\left(\left\|\sigma_{N}\right\|_{1 / 2, T \cap \Gamma_{C}}^{2}+\left\|u_{N}\right\|_{3 / 2, T \cap \Gamma_{C}}^{2}\right)
$$

Globally. When $\tau \in(3 / 2,2)$, the optimal choice of the value of $\theta$ to make a compromise between the estimates of the first and second cases (3.9) and (3.14) is $\theta=h^{2 \tau-3}$. This leads to

$$
\int_{\Gamma_{C}} \sigma_{N}\left(\mathcal{I}^{h} u\right)_{N} d \Gamma \leq C h^{2 \tau^{2}-5 \tau+4}\|u\|_{\tau, \Omega}^{2}
$$

which establishes (3.1). When $\tau=2$ we choose $q=2$ and $\theta=h^{2}$ in (3.10) and (3.15). So we obtain

$$
\int_{\Gamma_{C}} \sigma_{N}\left(\mathcal{I}^{h} u\right)_{N} d \Gamma \leq C h^{2} \ln (1 / h)\|u\|_{2, \Omega}^{2}
$$

from which estimate (3.2) follows.

4. An extension of the technique to another discrete contact condition. Let us again choose the $P_{1}$ finite element space $V^{h}$ defined in (2.8) and consider now the discrete contact condition incorporated in the closed convex cone $\overline{K^{h}}$ :

$$
\begin{aligned}
\overline{K^{h}} & =\left\{v^{h} \in V^{h}, \int_{T \cap \Gamma_{C}} v_{N}^{h} d \Gamma \leq 0 \forall T \in T^{h}\right\} \\
& =\left\{v^{h} \in V^{h}, v_{N}^{h}\left(\xi_{i}\right) \leq 0 \forall 1 \leq i \leq I\right\},
\end{aligned}
$$

where the $\xi_{i}$ are the midpoints of the $I$ contact segments (i.e., the segments $T \cap \Gamma_{C}$ of positive measure). Such a contact condition is classical when using hybrid methods involving Lagrange multipliers (see, e.g., [14, 19, 3]). Note that $\overline{K^{h}} \not \subset K$. The discrete variational inequality becomes

$$
\left\{\begin{array}{l}
\text { Find } \overline{u^{h}} \in \overline{K^{h}} \text { satisfying } \\
a\left(\overline{u^{h}}, v^{h}-\overline{u^{h}}\right) \geq l\left(v^{h}-\overline{u^{h}}\right) \quad \forall v^{h} \in \overline{K^{h}} .
\end{array}\right.
$$

According to Stampacchia's theorem, problem (4.1) admits a unique solution.

The specificity in the analysis of this problem comes from the fact that $\overline{K^{h}}$ is not a subset of $K$. As far as we know the existing results are the following: if $u \in H^{\tau}(\Omega)$ with $3 / 2<\tau \leq 2$, the analysis in $[14,19]$ leads to a convergence rate of order $h^{\tau / 2-1 / 4}$. (The existing results are of the same order as for the first approach using $K^{h}$ previously improved in this paper.) In particular an error bound of $h^{3 / 4}$ is obtained when $\tau=2$ (see $[14,19])$. Note also that a standard analysis gives a convergence of order $h$ when supposing that $u \in H^{5 / 2}(\Omega)$.

The forthcoming theorem gives improved a priori error estimates and is divided into three parts: a first part where the regularity of $u$ is assumed to lie between $H^{3 / 2}(\Omega)$ and $H^{2}(\Omega)$, a second part in which the $H^{2}(\Omega)$-regularity is considered separately, and a third result dealing with $H^{2+\varepsilon}(\Omega)$ regularity with $\varepsilon>0$. As previously, we denote by $C$ a positive generic constant which does not depend on either the mesh size $h$ or the solution $u$. 
THEOREM 4.1. Let $u$ and $\overline{u^{h}}$ be the solutions to problems (2.7) and (4.1), respectively.

Assume that $u \in\left(H^{\tau}(\Omega)\right)^{2}$ with $3 / 2<\tau<2$. Then, there exists a constant $C>0$ independent of $h$ and $u$ such that

$$
\left\|u-\overline{u^{h}}\right\|_{1, \Omega} \leq C h^{\tau^{2}-\frac{5}{2} \tau+2}\|u\|_{\tau, \Omega}
$$

Assume that $u \in\left(H^{2}(\Omega)\right)^{2}$. Then, there exists a constant $C>0$ independent of $h$ and $u$ such that

$$
\left\|u-\overline{u^{h}}\right\|_{1, \Omega} \leq C h \sqrt{|\ln (h)|}\|u\|_{2, \Omega} .
$$

Assume that $u \in\left(H^{2+\varepsilon}(\Omega)\right)^{2}$ for some $\varepsilon>0$. Then, there exists a constant $C>0$ independent of $h$ and $u$ such that

$$
\left\|u-\overline{u^{h}}\right\|_{1, \Omega} \leq C h\|u\|_{2+\varepsilon, \Omega} .
$$

Remark 4. Contrary to the previous approximation in (2.9), where the loss of optimality comes from the Lagrange interpolation operator which does not satisfy appropriate estimates in Sobolev norms with negative exponents, the loss of optimality in the case of approximation (4.1) comes from the $L^{2}$-projection operator on piecewise constant functions which does not approximate in a convenient way the functions that are more than $H^{1}$ regular.

Proof. The forthcoming analysis uses the same basic idea as in Theorem 3.1 but the technical details are quite different. The use of Falk's lemma in the case $\overline{K^{h}} \not \subset K$ gives (see, e.g., [3])

$$
\begin{aligned}
\left\|u-\overline{u^{h}}\right\|_{1, \Omega}^{2} \leq & C\left[\inf _{v^{h} \in \overline{K^{h}}}\left(\left\|u-v^{h}\right\|_{1, \Omega}^{2}+\int_{\Gamma_{C}} \sigma_{N}\left(v^{h}-u\right)_{N} d \Gamma\right)\right. \\
& \left.+\inf _{v \in K} \int_{\Gamma_{C}} \sigma_{N}\left(v-\overline{u^{h}}\right)_{N} d \Gamma\right] .
\end{aligned}
$$

As previously we can choose $v^{h}=\mathcal{I}^{h} u$, where $\mathcal{I}^{h}$ is the Lagrange interpolation operator mapping onto $V^{h}$ since $\mathcal{I}^{h} u \in K^{h} \subset \overline{K^{h}}$. The first infimum in (4.5) therefore satisfies the error bounds (4.2) and (4.3) in Theorem 4.1 according to Theorem 3.1. The first infimum in (4.5) satisfies also the bound of order $h$ in (4.4) when $H^{2+\varepsilon}$ regularity is assumed: this follows from the same analysis as in Theorem 3.1 by using estimate (5.3) (see also [23]).

In the second infimum in $(4.5)$, we choose $v=0$. To prove the theorem it remains then to estimate the term

$$
-\int_{\Gamma_{C}} \sigma_{N} \overline{u_{N}^{h}} d \Gamma .
$$

We next consider the space $X^{h}$ of the piecewise constant functions on the meshes of $T \cap \Gamma_{C}$

$$
X^{h}=\left\{\chi^{h} \in L^{2}\left(\Gamma_{C}\right):\left.\chi^{h}\right|_{T \cap \Gamma_{C}} ^{\in P_{0}}\left(T \cap \Gamma_{C}\right) \forall T \in T^{h}\right\}
$$

and the classical $L^{2}\left(\Gamma_{C}\right)$-projection operator $\pi^{h}: L^{2}\left(\Gamma_{C}\right) \rightarrow X^{h}$ defined for any $\varphi \in L^{2}\left(\Gamma_{C}\right)$ by

$$
\int_{\Gamma_{C}}\left(\varphi-\pi^{h} \varphi\right) \chi^{h} d \Gamma=0 \quad \forall \chi^{h} \in X^{h}
$$


We still denote by $h_{e}$ the length of the segment $T \cap \Gamma_{C}$. The operator $\pi^{h}$ satisfies the following estimates for any $0 \leq r \leq 1$ and any $\varphi \in H^{r}\left(\Gamma_{C}\right)$ (the proof is the same as the one in Lemma 6.1):

$$
\left\|\varphi-\pi^{h} \varphi\right\|_{0, T \cap \Gamma_{C}} \leq C h_{e}^{r}|\varphi|_{r, T \cap \Gamma_{C}} \quad \text { and } \quad\left\|\varphi-\pi^{h} \varphi\right\|_{0, \Gamma_{C}} \leq C h^{r}|\varphi|_{r, \Gamma_{C}} .
$$

When considering the dual norm $\|\cdot\|_{1 / 2, *, \Gamma_{C}}$ of $\|\cdot\|_{1 / 2, \Gamma_{C}}$ we deduce for any $0 \leq r \leq 1$ and any $\varphi \in H^{r}\left(\Gamma_{C}\right)$

$$
\begin{aligned}
\left\|\varphi-\pi^{h} \varphi\right\|_{1 / 2, *, \Gamma_{C}} & =\sup _{\psi \in H^{1 / 2}\left(\Gamma_{C}\right)} \frac{\int_{\Gamma_{C}}\left(\varphi-\pi^{h} \varphi\right) \psi d \Gamma}{\|\psi\|_{1 / 2, \Gamma_{C}}} \\
& \leq \sup _{\psi \in H^{1 / 2}\left(\Gamma_{C}\right)} \frac{\left\|\varphi-\pi^{h} \varphi\right\|_{0, \Gamma_{C}}\left\|\psi-\pi^{h} \psi\right\|_{0, \Gamma_{C}}}{\|\psi\|_{1 / 2, \Gamma_{C}}} \\
& \leq C h^{r+1 / 2}|\varphi|_{r, \Gamma_{C}} .
\end{aligned}
$$

We have, since $\pi^{h} \sigma_{N}$ is a nonpositive piecewise constant function on $\Gamma_{C}$,

$$
\begin{aligned}
-\int_{\Gamma_{C}} \sigma_{N} \overline{u_{N}^{h}} d \Gamma & \leq-\int_{\Gamma_{C}}\left(\sigma_{N}-\pi^{h} \sigma_{N}\right) \overline{u_{N}^{h}} d \Gamma \\
& =-\int_{\Gamma_{C}}\left(\sigma_{N}-\pi^{h} \sigma_{N}\right)\left(\overline{u_{N}^{h}}-u_{N}\right) d \Gamma-\int_{\Gamma_{C}}\left(\sigma_{N}-\pi^{h} \sigma_{N}\right) u_{N} d \Gamma .
\end{aligned}
$$

The first term in (4.8) is bounded in a optimal way by using (4.7), the trace theorem, and Young's inequality:

$$
\begin{aligned}
-\int_{\Gamma_{C}}\left(\sigma_{N}-\pi^{h} \sigma_{N}\right)\left(\overline{u_{N}^{h}}-u_{N}\right) d \Gamma & \leq\left\|\sigma_{N}-\pi^{h} \sigma_{N}\right\|_{1 / 2, *, \Gamma_{C}}\left\|\overline{u_{N}^{h}}-u_{N}\right\|_{1 / 2, \Gamma_{C}} \\
& \leq C h^{\tau-1}\left|\sigma_{N}\right|_{\tau-3 / 2, \Gamma_{C}}\left\|\overline{u^{h}}-u\right\|_{1, \Omega} \\
& \leq C h^{2(\tau-1)}\left|\sigma_{N}\right|_{\tau-3 / 2, \Gamma_{C}}^{2}+\frac{1}{2}\left\|u-\overline{u^{h}}\right\|_{1, \Omega}^{2} .
\end{aligned}
$$

To prove the theorem it remains now to bound the second term in (4.8). We estimate this term on any element $T \cap \Gamma_{C}$ :

$$
-\int_{T \cap \Gamma_{C}}\left(\sigma_{N}-\pi^{h} \sigma_{N}\right) u_{N} d \Gamma=\int_{T \cap \Gamma_{C}}\left(\sigma_{N}-\pi^{h} \sigma_{N}\right)\left(\pi^{h} u_{N}-u_{N}\right) d \Gamma .
$$

For any contact element $T \cap \Gamma_{C}$ we consider the (closed) set of contact points located on the segment $T \cap \overline{\Gamma_{C}}$ :

$$
C_{T}=\left\{x \in T \cap \overline{\Gamma_{C}} ; u_{N}(x)=0\right\} .
$$

Let $\max \left(d(x, y): x, y \in C_{T}\right)$ stand for the diameter of $C_{T}$, where $d(x, y)$ is the distance between $x$ and $y$. Let $0<\theta<1$ be fixed (the optimal choice of $\theta$ will be done later) and let $T \in T^{h}$ be an element such that $T \cap \Gamma_{C} \neq \emptyset$. We next consider the following alternative (which differs from the alternative leading to the result in Theorem 3.1), which is also depicted in Figure 4.1. 


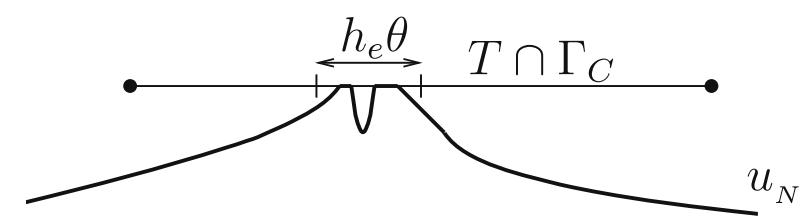

First case: the diameter of $C_{T}$ is smaller than $h_{e} \theta$.

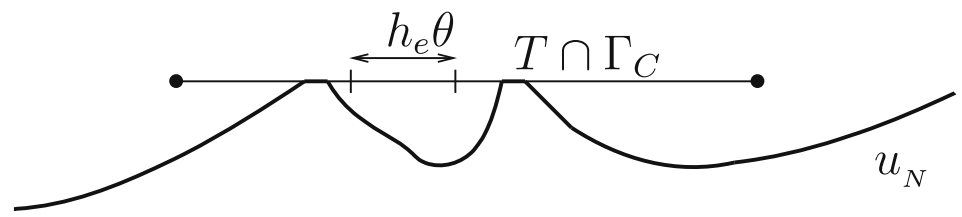

Second case: the diameter of $C_{T}$ is larger than $h_{e} \theta$.

FIG. 4.1. The alternative: first and second cases.

First case: the diameter of $C_{T}$ is smaller than $h_{e} \theta$.

Second case: the diameter of $C_{T}$ is larger than $h_{e} \theta$.

- For $3 / 2<\tau<2$.

First case. In this case the contact zone can be included into a segment $S$ of length $h_{e} \theta$ which means that $\sigma_{N}$ vanishes outside this segment. So

$$
\begin{aligned}
\int_{T \cap \Gamma_{C}}\left(\sigma_{N}-\pi^{h} \sigma_{N}\right)\left(\pi^{h} u_{N}-u_{N}\right) d \Gamma & =\int_{T \cap \Gamma_{C}} \sigma_{N}\left(\pi^{h} u_{N}-u_{N}\right) d \Gamma \\
& \leq\left\|\sigma_{N}\right\|_{L^{1}\left(T \cap \Gamma_{C}\right)}\left\|u_{N}-\pi^{h} u_{N}\right\|_{L^{\infty}\left(T \cap \Gamma_{C}\right)} \\
& \leq\left\|\sigma_{N}\right\|_{L^{1}\left(T \cap \Gamma_{C}\right)} h_{e}^{1 / 2}\left\|u_{N}^{\prime}\right\|_{0, T \cap \Gamma_{C}} \\
& =\left\|\sigma_{N}\right\|_{L^{1}(S)} h_{e}^{1 / 2}\left\|u_{N}^{\prime}\right\|_{0, T \cap \Gamma_{C}},
\end{aligned}
$$

where we use (6.2). The estimate of $\left\|\sigma_{N}\right\|_{L^{1}(S)}$ is handled exactly as $\left\|u_{N}^{\prime}\right\|_{L^{1}\left(a_{i}, \xi_{i}\right)}$ in (3.5) and we obtain

$$
\left\|\sigma_{N}\right\|_{L^{1}(S)} \leq C h_{e}^{1 / 2} \theta^{\tau-1}\left\|\sigma_{N}\right\|_{\tau-3 / 2, T \cap \Gamma_{C}}
$$

Combining both previous estimates yields

$$
\int_{T \cap \Gamma_{C}}\left(\sigma_{N}-\pi^{h} \sigma_{N}\right)\left(\pi^{h} u_{N}-u_{N}\right) d \Gamma \leq C h_{e} \theta^{\tau-1}\left\|\sigma_{N}\right\|_{\tau-3 / 2, T \cap \Gamma_{C}}\left\|u_{N}^{\prime}\right\|_{0, T \cap \Gamma_{C}}
$$

Second case. In this case there exist two contact points $c_{1}$ and $c_{2}$ such that $\left|c_{1}-c_{2}\right| \geq h_{e} \theta$ and we have

$$
\int_{c_{1}}^{c_{2}} u_{N}^{\prime}(x)=0
$$

which allows us to use estimate (5.1) together with Remark 7:

$$
\begin{aligned}
\int_{T \cap \Gamma_{C}}\left(\sigma_{N}-\pi^{h} \sigma_{N}\right)\left(\pi^{h} u_{N}-u_{N}\right) d \Gamma & \leq\left\|\sigma_{N}-\pi^{h} \sigma_{N}\right\|_{0, T \cap \Gamma_{C}}\left\|u_{N}-\pi^{h} u_{N}\right\|_{0, T \cap \Gamma_{C}} \\
& \leq C h_{e}^{\tau-1 / 2}\left|\sigma_{N}\right|_{\tau-3 / 2, T \cap \Gamma_{C}}\left\|u_{N}^{\prime}\right\|_{0, T \cap \Gamma_{C}} \\
& \leq C h_{e}^{2 \tau-2} \theta^{\tau-2}\left|\sigma_{N}\right|_{\tau-3 / 2, T \cap \Gamma_{C}}\left|u_{N}^{\prime}\right|_{\tau-3 / 2, T \cap \Gamma_{C}},
\end{aligned}
$$

where we use (4.6) and the same estimate as in (3.12) to bound $\left\|u_{N}^{\prime}\right\|_{0, T \cap \Gamma_{C}}$. 
Globally. In (4.10) and (4.11) we choose $\theta=h^{2 \tau-3}$, which yields

$$
\int_{\Gamma_{C}}\left(\sigma_{N}-\pi^{h} \sigma_{N}\right)\left(\pi^{h} u_{N}-u_{N}\right) d \Gamma \leq C h^{2 \tau^{2}-5 \tau+4}\|u\|_{\tau, \Omega}^{2}
$$

and gives the result in (4.2).

- For $\tau=2$, the same method leads to the following estimates.

First case: The same approach as in (4.10) is chosen by using for any $q>1$ the continuous embedding of $H^{1 / 2}\left(T \cap \Gamma_{C}\right)$ into $L^{q}\left(T \cap \Gamma_{C}\right)$. So, for any $q>1$, we get

$$
\int_{T \cap \Gamma_{C}}\left(\sigma_{N}-\pi^{h} \sigma_{N}\right)\left(\pi^{h} u_{N}-u_{N}\right) d \Gamma \leq C(q) h_{e} \theta^{1-\frac{1}{q}}\left\|\sigma_{N}\right\|_{1 / 2, T \cap \Gamma_{C}}\left\|u_{N}^{\prime}\right\|_{0, T \cap \Gamma_{C}}
$$

Second case: Achieving the same calculations as in (4.11) and using (5.2), we get

$$
\int_{T \cap \Gamma_{C}}\left(\sigma_{N}-\pi^{h} \sigma_{N}\right)\left(\pi^{h} u_{N}-u_{N}\right) d \Gamma \leq C h_{e}^{2} \ln (1 / \theta)\left|\sigma_{N}\right|_{1 / 2, T \cap \Gamma_{C}}\left|u_{N}^{\prime}\right|_{1 / 2, T \cap \Gamma_{C}}
$$

Globally. In (4.12), (4.13) we choose $q=2$ and $\theta=h^{2}$. So we obtain

$$
\int_{\Gamma_{C}}\left(\sigma_{N}-\pi^{h} \sigma_{N}\right)\left(\pi^{h} u_{N}-u_{N}\right) d \Gamma \leq C h^{2} \ln (1 / h)\|u\|_{2, \Omega}^{2}
$$

which gives the result in (4.3).

- For $\tau=2+\varepsilon(\varepsilon>0$ can be supposed arbitrarily small), the situation is simpler. Either there is no contact point on $T \cap \Gamma_{C}$ and the term (4.9) vanishes or there exists a contact point and the same calculations as in (4.11) (or (4.13)) using (5.3) lead to the bound

$$
\int_{T \cap \Gamma_{C}}\left(\sigma_{N}-\pi^{h} \sigma_{N}\right)\left(\pi^{h} u_{N}-u_{N}\right) d \Gamma \leq C h_{e}^{2+2 \varepsilon}\left|\sigma_{N}\right|_{1 / 2+\varepsilon, T \cap \Gamma_{C}}\left|u_{N}^{\prime}\right|_{1 / 2+\varepsilon, T \cap \Gamma_{C}}
$$

As a consequence

$$
\int_{\Gamma_{C}}\left(\sigma_{N}-\pi^{h} \sigma_{N}\right)\left(\pi^{h} u_{N}-u_{N}\right) d \Gamma \leq C h^{2+2 \varepsilon}\|u\|_{2+\varepsilon, \Omega}^{2}
$$

which leads to the result in (4.4).

5. Appendix A: Estimate for some Poincaré constants. The use of Poincaré inequalities is a key tool to obtain the estimates of the "second cases" in (3.14), (3.15), (4.11), (4.13), and (4.14). In these estimates $\sigma_{N}$ or $u_{N}^{\prime}$ is supposed to vanish on a area of length at least $h_{e} \theta$. So we need to estimate precisely the constant $C$ as a function of the length of the vanishing area. Note that there is a close link between the determination of these constants and Bessel's theory of capacity (see, e.g., [31]). We do not use the tools of this theory and all the proofs concerning Poincaré constants are made independently using scaling arguments to render the paper self-contained.

For $u \in H^{\nu}(0,1)$ and $0<\nu<1$ we denote by

$$
|u|_{\nu,(0,1)}=\left(\int_{0}^{1} \int_{0}^{1} \frac{(u(x)-u(y))^{2}}{|x-y|^{1+2 \nu}} d x d y\right)^{1 / 2}
$$


the classical seminorm and we recall that (see (1.1))

$$
\|u\|_{\nu,(0,1)}=\left(\|u\|_{0,(0,1)}^{2}+|u|_{\nu,(0,1)}^{2}\right)^{1 / 2}
$$

Let us first recall the Peetre-Tartar lemma, which is a standard tool to establish Poincaré inequalities (see, e.g., [22, 26, 27, 7]).

Lemma 5.1 (Peetre-Tartar). Let $X, Y, Z$ be three Banach spaces. Let $A \in$ $\mathcal{L}(X, Y)$ be injective and let $T \in \mathcal{L}(X, Z)$ be compact. If there exists a constant $c>0$ such that for all $x \in X, c\|x\|_{X} \leq\|A x\|_{Y}+\|T x\|_{Z}$, then there exists $\alpha>0$ such that for all $x \in X$

$$
\alpha\|x\|_{X} \leq\|A x\|_{Y}
$$

The following result concerns the estimate of the Poincaré constant on the interval $\tilde{I}=(0,1)$ for the functions in $H^{\nu}(\tilde{I}), 0<\nu<1$, with respect to the length of the interval on which the mean of the function vanishes.

LemMa 5.2. Let $0<\nu<1, \tilde{I}=(0,1), 0<\theta<1$, and $\tilde{u} \in H^{\nu}(\tilde{I})$, satisfying $\int_{0}^{\theta} \tilde{u}(x) d x=0$. There exist constants $C=C(\nu)>0$ independent of $\tilde{u}$ and $\theta$ such that - if $0<\nu<1 / 2$, then

$$
\|\tilde{u}\|_{0, \tilde{I}} \leq C \theta^{\nu-1 / 2}|\tilde{u}|_{\nu, \tilde{I}}
$$

- if $\nu=1 / 2$, then

$$
\|\tilde{u}\|_{0, \tilde{I}} \leq C \ln (1 / \theta)|\tilde{u}|_{\nu, \tilde{I}}
$$

- if $1 / 2<\nu<1$, then

$$
\|\tilde{u}\|_{0, \tilde{I}} \leq C|\tilde{u}|_{\nu, \tilde{I}}
$$

Remark 5. It is easy to show that estimate (5.3) does not hold when $\nu=1 / 2$. Consider a nonnegative function $\tilde{u} \in H^{1 / 2}(\tilde{I})$ which is not in $L^{\infty}(\tilde{I})$ (e.g., $\tilde{u}(x)=$ $|\ln (x)|^{\alpha}$ with $\left.0<\alpha<1 / 2\right)$ and suppose without loss of generality that $\|\tilde{u}\|_{1 / 2, \tilde{I}}=1$. Define the truncated functions $\left(\tilde{u}_{n}\right)_{n}=\min (\tilde{u}, n)$. Therefore $\left\|\tilde{u}_{n}\right\|_{L^{\infty}(\tilde{I})}=n$ and $\left\|\tilde{u}_{n}\right\|_{1 / 2, \tilde{I}} \leq\|\tilde{u}\|_{1 / 2, \tilde{I}}=1$. Let $\tilde{v}_{n}=\tilde{u}_{n} / n$; then $\left\|\tilde{v}_{n}\right\|_{L^{\infty}(\tilde{I})}=1$ and $\left\|\tilde{v}_{n}\right\|_{1 / 2, \tilde{I}} \leq 1 / n$. Set finally $\tilde{w}_{n}=1-\tilde{v}_{n} ; \tilde{w}_{n}$ vanishes on a small interval and $\left\|\tilde{w}_{n}\right\|_{L^{2}(\tilde{I})} \geq\|1\|_{L^{2}(\tilde{I})}-$ $\left\|\tilde{v}_{n}\right\|_{L^{2}(\tilde{I})} \geq 1-1 / n$, whereas $\left|\tilde{w}_{n}\right|_{1 / 2, \tilde{I}} \leq\left\|\tilde{w}_{n}\right\|_{1 / 2, \tilde{I}}=\left\|\tilde{v}_{n}\right\|_{1 / 2, \tilde{I}} \leq 1 / n$.

Proof. Let us consider the following closed subspace of $H^{\nu}(\tilde{I})$ :

$$
V_{\theta}=\left\{\tilde{v} \in H^{\nu}(\tilde{I}): \int_{0}^{\theta} \tilde{v}(x) d x=0\right\} .
$$

One can apply the Peetre-Tartar lemma for $X=V_{\theta}, Y=L^{2}(\tilde{I} \times \tilde{I}), Z=L^{2}(\tilde{I})$, $A: \tilde{u} \mapsto A \tilde{u}$ such that $A \tilde{u}(x, y)=(\tilde{u}(x)-\tilde{u}(y)) /|x-y|^{1 / 2+\nu}$ and $T$ is the compact embedding operator from $X$ into $Z$. The operator $A$ is injective since $A \tilde{u}=0$ implies that $\tilde{u}$ is a.e. a constant and the only constant of $V_{\theta}$ is 0 . Consequently, there exists a constant $\gamma>0$ such that

$$
\|\tilde{u}\|_{0, \tilde{I}} \leq \gamma|\tilde{u}|_{\nu, \tilde{I}} \quad \forall \tilde{u} \in V_{\theta}
$$


In the following, we denote by $\gamma_{\theta}$ the best constant satisfying this inequality in (5.4). The proof of the estimate of $\gamma_{\theta}$ as a function of $\theta$ consists in a scaling argument. Let $\tilde{u} \in H^{\nu}(\tilde{I})$; consider now the interval $I=(0,1 / \theta)$. Denoting

$$
u(x)=\tilde{u}(\theta x)
$$

for any $x \in(0,1 / \theta)$, we have $u \in H^{\nu}(I)$, and an elementary calculation leads to

$$
\|u\|_{0, I}=\theta^{-1 / 2}\|\tilde{u}\|_{0, \tilde{I}}, \quad|u|_{\nu, I}=\theta^{\nu-1 / 2}|\tilde{u}|_{\nu, \tilde{I}} .
$$

Denoting by $c=\int_{0}^{1} u(x) d x=\theta^{-1} \int_{0}^{\theta} \tilde{u}(x) d x$ the mean value of $\tilde{u}$ on $[0, \theta]$, observing that $\tilde{u}-c \in V_{\theta}$, one obtains thanks to (5.4) and (5.5)

$$
\|u-c\|_{0, I}=\theta^{-1 / 2}\|\tilde{u}-c\|_{0, \tilde{I}} \leq \theta^{-1 / 2} \gamma_{\theta}|\tilde{u}|_{\nu, \tilde{I}}=\theta^{-\nu} \gamma_{\theta}|u|_{\nu, I}
$$

and consequently

$$
\|u\|_{0, I} \leq\|c\|_{0, I}+\|u-c\|_{0, I} \leq \theta^{-1 / 2}|c|+\theta^{-\nu} \gamma_{\theta}|u|_{\nu, I} .
$$

Suppose now that $u$ satisfies $\int_{0}^{\theta} u(x) d x=0$; one obtains (since $\left.u\right|_{\tilde{I}} \in V_{\theta}$ )

$$
|c| \leq\|u\|_{0, \tilde{I}} \leq \gamma_{\theta}|u|_{\nu, \tilde{I}} \leq \gamma_{\theta}|u|_{\nu, I} .
$$

From (5.6) and (5.7), we deduce

$$
\|u\|_{0, I} \leq \gamma_{\theta}\left(\theta^{-1 / 2}+\theta^{-\nu}\right)|u|_{\nu, I} .
$$

The latter bound allows to obtain the following estimate for $\tilde{u} \in V_{\theta^{2}}$ :

$$
\|\tilde{u}\|_{0, \tilde{I}}=\theta^{1 / 2}\|u\|_{0, I} \leq \gamma_{\theta}\left(1+\theta^{1 / 2-\nu}\right)|u|_{\nu, I}=\gamma_{\theta}\left(1+\theta^{\nu-1 / 2}\right)|\tilde{u}|_{\nu, \tilde{I}} .
$$

With this method, we obtain an estimate of the evolution of the Poincaré constant when the length of the zone on which the mean of $\tilde{u}$ vanishes varies from $\theta$ to $\theta^{2}$ :

$$
\gamma_{\theta^{2}} \leq \gamma_{\theta}\left(1+\theta^{\nu-1 / 2}\right)
$$

By induction from (5.8), the Poincaré constant for a zone of length $\theta^{2^{n}}$ on which the mean of $\tilde{u}$ vanishes is

$$
\begin{aligned}
\gamma_{\theta^{2}} & \leq \gamma_{\theta}\left(1+\theta^{\nu-1 / 2}\right)\left(1+\theta^{2(\nu-1 / 2)}\right) \cdots\left(1+\theta^{2^{n-1}(\nu-1 / 2)}\right) \\
& =\gamma_{\theta} \prod_{i=0}^{n-1}\left(1+\theta^{2^{i}(\nu-1 / 2)}\right) .
\end{aligned}
$$

Now, we fix $\theta_{0} \in(0,1)$ (e.g., $\left.\theta_{0}=1 / 2\right)$. If $\theta \in\left(0, \theta_{0}\right)$, there exists $n \in \mathbb{N}$ such that $\theta_{0}^{2^{n+1}} \leq \theta \leq \theta_{0}^{2^{n}}$ (hence $\left.2^{n} \leq \ln (\theta) / \ln \left(\theta_{0}\right)\right)$, so $\gamma_{\theta_{0}^{2^{n}}} \leq \gamma_{\theta} \leq \gamma_{\theta_{0}^{2 n+1}}$. The three cases of the lemma are handled as follows by using (5.9):

- If $\nu=1 / 2$ this gives

$$
\gamma_{\theta} \leq \gamma_{\theta_{0}^{2 n+1}} \leq 2^{n+1} \gamma_{\theta_{0}} \leq 2 \frac{\gamma_{\theta_{0}}}{\ln \left(\theta_{0}\right)} \ln (\theta)=2 \frac{\gamma_{\theta_{0}}}{\ln \left(1 / \theta_{0}\right)} \ln (1 / \theta)=C\left(\gamma_{\theta_{0}}, \theta_{0}\right) \ln (1 / \theta),
$$

which proves (5.2). 
- If $1 / 2<\nu<1$, one has (using the estimate $\ln (1+x) \leq x$ for $x \geq 0$ )

$$
\begin{aligned}
\ln \left(\gamma_{\theta}\right) \leq \ln \left(\gamma_{\theta_{0}^{2 n+1}}\right) \leq \ln \left(\gamma_{\theta_{0}}\right)+\sum_{i=0}^{n} \ln \left(1+\theta_{0}^{2^{i}(\nu-1 / 2)}\right) & \leq \ln \left(\gamma_{\theta_{0}}\right)+\sum_{i=0}^{n}\left(\theta_{0}^{(\nu-1 / 2)}\right)^{2^{i}} \\
& \leq C\left(\gamma_{\theta_{0}}, \theta_{0}, \nu\right)
\end{aligned}
$$

with $C\left(\gamma_{\theta_{0}}, \theta_{0}, \nu\right)$ independent of $n$ (since $0<\theta_{0}^{\nu-1 / 2}<1$ ), which implies that $\gamma_{\theta}$ is bounded by a constant independent of $\theta$ and leads to estimate (5.3).

- If $0<\nu<1 / 2$, we need to achieve a more precise analysis than in the first two cases. As previously mentioned we have $0<\theta<\theta_{0}<1$ and there exists $n \in \mathbb{N}$ such that $\theta_{0}^{2^{n+1}} \leq \theta \leq \theta_{0}^{2^{n}}$ or equivalently $\theta=\theta_{0}^{\alpha 2^{n}}$ with $1 \leq \alpha \leq 2$. Consequently, setting $\bar{\theta}=\theta_{0}{ }^{\alpha / 2}$ we have $\theta_{0} \leq \bar{\theta} \leq \theta_{0}^{1 / 2}<1$ (hence $\gamma_{\bar{\theta}} \leq \gamma_{\theta_{0}}$ ) and $\theta=\bar{\theta}^{2^{n+1}}$. One has (using again the estimate $\ln (1+x) \leq x$ for $x \geq 0)$

$$
\begin{aligned}
\ln \left(\gamma_{\theta}\right)=\ln \left(\gamma_{\bar{\theta}^{2 n+1}}\right) & \leq \ln \left(\gamma_{\bar{\theta}}\right)+\sum_{i=0}^{n} \ln \left(1+\bar{\theta}^{2^{i}(\nu-1 / 2)}\right) \\
& \leq \ln \left(\gamma_{\bar{\theta}}\right)+\sum_{i=0}^{n}\left(\ln \left(\bar{\theta}^{2^{i}(\nu-1 / 2)}\right)+\ln \left(1+\bar{\theta}^{2^{i}(1 / 2-\nu)}\right)\right) \\
& \leq \ln \left(\gamma_{\bar{\theta}}\right)+\ln \left(\bar{\theta}^{\left(2^{n+1}-1\right)(\nu-1 / 2)}\right)+\sum_{i=0}^{n}\left(\bar{\theta}^{1 / 2-\nu}\right)^{2^{i}} \\
& \leq \ln \left(\gamma_{\theta_{0}}\right)+(1 / 2-\nu) \ln (1 / \theta)+\sum_{i=0}^{n}\left(\theta_{0}^{1 / 4-\nu / 2}\right)^{2^{i}} .
\end{aligned}
$$

Hence

$$
\gamma_{\theta} \leq C\left(\gamma_{\theta_{0}}, \theta_{0}, \nu\right) \theta^{\nu-1 / 2}
$$

where $C\left(\gamma_{\theta_{0}}, \theta_{0}, \nu\right)$ is a positive constant depending only on $\gamma_{\theta_{0}}, \theta_{0}$, and $\nu$. This concludes the proof.

Remark 6 . The space $H^{\nu}(\tilde{I})$ is compactly included into $C^{0}(\tilde{I})$ for $\nu>1 / 2$ so that the Poincaré inequality is valid for functions vanishing at a single point of $\tilde{I}$. (This is a direct consequence of the Peetre-Tartar lemma; see [23].)

Remark 7. It is easy to check that the constants $C(\theta)$ obtained in Lemma 5.2 (i.e., $C(\theta)=C \theta^{\nu-1 / 2}$ if $0<\nu<1 / 2, C(\theta)=C \ln (1 / \theta)$ if $\nu=1 / 2$, and $C(\theta)=C$ if $1 / 2<\nu<1$ ) are still valid independently on the location of the set (of length $\theta$ ) where the average of $\tilde{u}$ vanishes. Suppose that $\int_{a}^{a+\theta} \tilde{u}(x) d x=0$ with $0<a<a+\theta<1$ and set $\tilde{I}_{1}=(0, a /(1-\theta))$ and $\tilde{I}_{2}=(a /(1-\theta), 1)$. Denoting by $\left|\tilde{I}_{1}\right|$ (resp., $\left.\left|\tilde{I}_{2}\right|\right)$ the length of $\tilde{I}_{1}$ (resp., $\tilde{I}_{2}$ ), passing on the reference element and according to the Lemma 5.2 we have

$$
\begin{aligned}
\|\tilde{u}\|_{0, \tilde{I}}^{2} & =\|\tilde{u}\|_{0, \tilde{I}_{1}}^{2}+\|\tilde{u}\|_{0, \tilde{I}_{2}}^{2} \\
& \leq\left(C(\theta)\left|\tilde{I}_{1}\right|^{\nu}|\tilde{u}|_{\nu, \tilde{I}_{1}}\right)^{2}+\left(C(\theta)\left|\tilde{I}_{2}\right|^{\nu}|\tilde{u}|_{\nu, \tilde{I}_{2}}\right)^{2} \\
& \leq(C(\theta))^{2}|\tilde{u}|_{\nu, \tilde{I}}^{2}
\end{aligned}
$$


6. Appendix B. Some interpolation error estimates in fractional order Hilbert spaces. In this appendix we denote by $\mathcal{I}^{h}$ the Lagrange interpolation operator of degree one in one dimension. (Note that we still choose the notation $\mathcal{I}^{h}$ in section 3 to denote the Lagrange interpolation operator of degree one in the two-dimensional space.) If $\nu \in(0,1)$ and $I$ stands for an interval, we set

$$
|u|_{1+\nu, I}=\left(\int_{I} \int_{I} \frac{\left(u^{\prime}(x)-u^{\prime}(y)\right)^{2}}{|x-y|^{1+2 \nu}} d x d y\right)^{1 / 2} .
$$

According to (1.1), the previous expression equals $\left|u^{\prime}\right|_{\nu, I}$. The following lemma deals with error estimates for $u-\mathcal{I}^{h} u$ when $u$ lies in fractional order Hilbert spaces. (The case of standard Hilbert spaces is well known; see, e.g., [5].) Note that the same kind of interpolation error estimate can be found, for instance, in [6]. The proof of the result we need is given here for the self-consistency of the paper.

Lemma 6.1 (local estimate). Let $I=(a, b)$ with $|b-a|=h>0$ and $0<\nu<1$. Then for $u \in H^{1+\nu}(I)$, we have

$$
\begin{gathered}
\left\|u-\mathcal{I}^{h} u\right\|_{0, I} \leq h^{1+\nu}|u|_{1+\nu, I}, \\
\left\|u-\mathcal{I}^{h} u\right\|_{1, I} \leq h^{\nu}|u|_{1+\nu, I} .
\end{gathered}
$$

Proof. One obtains, by an elementary calculation (since $\left.\left(u-\mathcal{I}^{h} u\right)(a)=0\right)$,

$$
\left\|u-\mathcal{I}^{h} u\right\|_{0, I} \leq h\left\|\left(u-\mathcal{I}^{h} u\right)^{\prime}\right\|_{0, I}=h\left\|u^{\prime}-\overline{u^{\prime}}\right\|_{0, I},
$$

where $\overline{u^{\prime}}=(u(b)-u(a)) /(b-a)$ denotes the mean value of $u^{\prime}$ on $I$. Let $v \in H^{\nu}(I)$ and denote by $\bar{v}$ its mean value on $I$. For any $x \in I$, we get

$$
\begin{aligned}
v(x)-\bar{v} & =h^{-1} \int_{I} v(x)-v(y) d y \\
& =h^{-1} \int_{I} \frac{v(x)-v(y)}{|x-y|^{\frac{1+2 \nu}{2}}}|x-y|^{\frac{1+2 \nu}{2}} d y .
\end{aligned}
$$

Note that when $x \in I$ and $\nu=1$ we have

$$
v(x)-\bar{v}=h^{-1} \int_{I} v(x)-v(y) d y=h^{-1} \int_{I} \int_{y}^{x} v^{\prime}(t) d t d y .
$$

Hence

$$
|v(x)-\bar{v}| \leq h^{\frac{1}{2}}\left\|v^{\prime}\right\|_{0, I} .
$$

Using the Cauchy-Schwarz inequality in estimate (6.1) we deduce

$$
\begin{aligned}
\int_{I}(v(x)-\bar{v})^{2} d x & =h^{-2} \int_{I}\left(\int_{I} \frac{v(x)-v(y)}{|x-y|^{\frac{1+2 \nu}{2}}}|x-y|^{\frac{1+2 \nu}{2}} d y\right)^{2} d x \\
& \leq h^{-2} \int_{I}\left(\int_{I} \frac{(v(x)-v(y))^{2}}{|x-y|^{1+2 \nu}} d y \int_{I}|x-y|^{1+2 \nu} d y\right) d x \\
& \leq h^{2 \nu} \int_{I} \int_{I} \frac{(v(x)-v(y))^{2}}{|x-y|^{1+2 \nu}} d y d x \\
& =h^{2 \nu}|v|_{\nu, I}^{2} .
\end{aligned}
$$


Changing $v$ with $u^{\prime}$ yields the result. The same calculation on $\left\|u-\mathcal{I}^{h} u\right\|_{1, I}$ leads to the second bound.

Lemma 6.2 (global estimate). Let $I^{h}$ be a mesh of a one-dimensional domain $\Gamma$. Then the following estimate holds for $u \in H^{1+\nu}(\Gamma), 0<\nu<1$ :

$$
\begin{gathered}
\left\|u-\mathcal{I}^{h} u\right\|_{0, \Gamma} \leq h^{1+\nu}|u|_{1+\nu, \Gamma}, \\
\left\|u-\mathcal{I}^{h} u\right\|_{1, \Gamma} \leq h^{\nu}|u|_{1+\nu, \Gamma}
\end{gathered}
$$

where $h$ is the size of the largest element of $I^{h}$.

Proof. By the previous lemma, one has

$$
\left\|u-\mathcal{I}^{h} u\right\|_{0, \Gamma}^{2}=\sum_{I \in I^{h}}\left\|u-\mathcal{I}^{h} u\right\|_{0, I}^{2} \leq \sum_{I \in I^{h}} h^{2+2 \nu}|u|_{1+\nu, I}^{2} \leq h^{2+2 \nu}|u|_{1+\nu, \Gamma}^{2} .
$$

The same calculation on $\left\|u-\mathcal{I}^{h} u\right\|_{1, \Gamma}$ leads to the second result.

7. Conclusion. In this paper we presented a new technique in order to improve the existing convergence rates for the two-dimensional Signorini problem approximated by the linear finite element method. The extension of this technique to other nonlinear problems, in particular free boundary problems, or to other approximation methods or to three-dimensional problems could be considered.

\section{REFERENCES}

[1] R. A. Adams, Sobolev Spaces, Academic Press, New York, 1975.

[2] F. Ben Belgacem, Numerical simulation of some variational inequalities arisen from unilateral contact problems by the finite element method, SIAM J. Numer. Anal., 37 (2000), pp. 1198-1216.

[3] F. Ben Belgacem and Y. Renard, Hybrid finite element methods for the Signorini problem, Math. Comp., 72 (2003), pp. 1117-1145.

[4] F. Brezzi, W. W. Hager, AND P. A. Raviart, Error estimates for the finite element solution of variational inequalities, Numer. Math., 28 (1977), pp. 431-443.

[5] P. G. Ciarlet, The finite element method for elliptic problems, in Handbook of Numerical Analysis, vol. 2, part 1, P. G. Ciarlet and J.-L. Lions, eds., North-Holland, Amsterdam, 1991, pp. 17-352.

[6] T. Dupont And R. Scott, Polynomial approximation of functions in Sobolev spaces, Math. Comp., 34 (1980), pp. 441-463.

[7] A. Ern and J.-L. Guermond, Theory and Practice of Finite Elements, Appl. Math. Sci. 159, Springer-Verlag, Berlin, 2004.

[8] R. S. FALK, Error estimates for the approximation of a class of variational inequalities, Math. Comp., 28 (1974), pp. 963-971.

[9] G. FicherA, Problemi elastostatici con vincoli unilaterali: il problema di Signorini con ambigue condizioni al contorno, Atti Accad. Naz. Lincei Mem. Cl. Sci. Fis. Mat. Natur. Sez., I (8) 7 (1963/1964), pp. 91-140.

[10] P. Grisvard, Elliptic Problems in Nonsmooth Domains, Monogr. Stud. Math. 24, Pitman, Boston, 1985.

[11] J. HASLINGER, Finite element analysis for unilateral problems with obstacles on the boundary, Aplikace Matematiky, 22 (1977), pp. 180-188.

[12] J. Haslinger And I. HlaváČEK, Contact between elastic bodies: 1. Continuous problems, Aplikace Matematiky, 25 (1980), pp. 324-347.

[13] J. Haslinger And I. HlavÁČEK, Contact between elastic bodies: 2. Finite element analysis, Aplikace Matematiky, 26 (1981), pp. 263-290.

[14] J. HASLinger, AND I. HlavÁČEK, AND J. NeČAs, Numerical methods for unilateral problems in solid mechanics, in Handbook of Numerical Analysis, vol. 4, part 2, P. G. Ciarlet and J.-L. Lions, eds., North-Holland, Amsterdam, 1996, pp. 313-485. 
[15] P. HiLd, A propos d'approximation par éléments finis optimale pour les problèmes de contact unilatéral, C. R. Acad. Sci. Paris Sér. I, 326 (1998), pp. 1233-1236.

[16] S. Hüeber And B. Wohlmuth, An optimal a priori error estimate for nonlinear multibody contact problems, SIAM J. Numer. Anal., 43 (2005), pp. 156-173.

[17] N. Kikuchi and J. T. Oden, Contact Problems in Elasticity, SIAM, Philadelphia, 1988.

[18] T. Laursen, Computational Contact and Impact Mechanics, Springer-Verlag, Berlin, 2002.

[19] K. Lhalouani And T. Sassi, Nonconforming mixed variational formulation and domain decomposition for unilateral problems, East-West J. Numer. Math., 7 (1999), pp. 23-30.

[20] J.-L. Lions and E. Magenes, Problèmes aux limites non homogènes, Dunod, Paris, 1968.

[21] M. Moussaoui And K. Khodja, Régularité des solutions d'un problème mêlé DirichletSignorini dans un domaine polygonal plan, Comm. Partial Differential Equations, 17 (1992), pp. 805-826.

[22] J. PeEtre, Espaces d'interpolation et Théorème de Sobolev, Ann. Inst. Fourier, 6 (1966), pp. 279-317.

[23] Y. RenaRd, A quasi-optimal a priori error estimate for Signorini's problem approximated by linear finite elements, C. R. Acad. Sci. Paris Séri. I, 350 (2012), pp. 325-328.

[24] F. Scarpini And M. A. Vivaldi, Approximation of an elliptic boundary value problem with unilateral constraints, RAIRO Anal. Numer., 11 (1977), pp. 197-208.

[25] A. Signorini, Questioni di elasticità non linearizzata e semilinearizzata, Rend. Mat. Appl., (5) 18 (1959), pp. 95-139.

[26] L. TartaR, Nonlinear Partial Differential Equations Using Compactness Methods, Report 1584, Mathematics Research Center, University of Wisconsin, Madison, 1975.

[27] L. TARTAR, Sur un lemme d'équivalence utilisé en analyse numérique, Calcolo, 24 (1987), pp. 129-140.

[28] H. Triebel, Interpolation Theory, Function Spaces, Differential Operators, North-Holland, Amsterdam, 1978.

[29] B. Wohlmuth, Variationally consistent discretization schemes and numerical algorithms for contact problems, Acta Numer., 20 (2011), pp. 569-734.

[30] P. Wriggers, Computational Contact Mechanics, Wiley, New York, 2002.

[31] W. P. Ziemer, Weakly Differentiable Functions, Springer, Berlin, 1989. 